\title{
Role of the cardiac nerve in the adaptive changes of heart rate in response to an aversive stimulus in Megalobulimus mogianensis
}

\author{
S.M.B. Romero ${ }^{1}$ and A. Hoffmann² \\ ${ }^{1}$ Departamento de Biologia, Faculdade de Filosofia, Ciências e Letras de Ribeirão Preto, ${ }^{2}$ Departamento \\ de Fisiologia, Faculdade de Medicina de Ribeirão Preto, Universidade de São Paulo, Ribeirão Preto, SP, \\ Brasil
}

Correspondence to: S.M.B. Romero, Departamento de Biologia, FFCLRP, USP, Av. Bandeirantes, 3900, 14040-901 Ribeirão Preto, SP, Brasil

Fax: +55-16-3602-3666. E-mail: sbromero@ffclrp.usp.br

The effect of an aversive stimulus represented by contact with a hot plate on the heart rate of Megalobulimus mogianensis was evaluated with electrocardiogram recording in intact snails $(\mathrm{N}=8)$. All stimulated animals showed an increase in heart rate, with mean values ranging from $35.6 \pm 1.2$ (basal heart rate) to $43.8 \pm 0.9 \mathrm{bpm}$ (post-stimulation heart rate). The cardioacceleration was followed by gradual recovery of the basal heart rate, with mean recovery times varying from $4.3 \pm 0.3$ to $5.8 \pm 0.6 \mathrm{~min}$. Repetition of the stimulus did not affect the magnitude of variation nor did it influence the basal heart rate recovery time. To investigate the role of the cardiac nerve in mediating the heart rate alterations induced by the aversive stimulus, denervated $(\mathrm{N}$ $=8$ ) and sham-operated $(\mathrm{N}=8)$ animals were also tested. Although the aversive stimulus caused the heart rate to increase significantly in both experimental groups, the mean increase in heart rate in denervated animals $(4.4 \pm 0.4 \mathrm{bpm})$ was $57 \%$ of the value obtained in sham-operated animals $(7.7 \pm 1.3 \mathrm{bpm})$, indicating that the cardiac nerve is responsible for $43 \%$ of the cardioacceleration induced by the aversive stimulus. The cardioacceleration observed in denervated snails may be due to an increase in venous return promoted by the intense muscular activity associated with the withdrawal response. Humoral factors may also be involved. A probable delaying inhibitory effect of the cardiac nerve on the recuperation of the basal heart rate is suggested.

Key words: Aversive stimulus; Cardiac nerve; Heart rate; Snail; Megalobulimus mogianensis

Publication supported by FAPESP.

Received November 27, 2007. Accepted May 6, 2008

All animals have the capacity to react to sudden environmental stimuli. In snails, innocuous or noxious stimulation applied to any exposed skin area usually produces an immediate withdrawal response. Previous investigations conducted in our laboratory revealed that specimens of Megalobulimus mogianensis placed on a hot aluminum plate at $52^{\circ} \mathrm{C}$ show stereotyped avoidance behavior consisting of characteristic elevation of the anterior portion of the body (1).

Withdrawal reflexes are associated with intense mus- cular activity. Since mollusks have an open circulatory system and, therefore, large hemocoelic sinuses, the contraction of body wall musculature squeezes the hemolymph and indirectly affects cardiac activity. Consequently, behavioral responses are, in general, associated with concomitant changes in heart rate (2-4). Moreover, cardiac activity is controlled in part by the nervous system $(3,4)$ and is a sensitive indicator of the animal's perception of any changes in its immediate surroundings (5).

A number of investigators have studied different as- 
pects of the withdrawal response evoked by a novel, innocuous or noxious stimulus $(1,4,6,7)$. Although the pathways that control defensive behaviors often share circuitry with those that control cardiovascular function (8), literature on heart rate alterations associated with these behaviors is scarce. The effects of a strong mechanical stimulation of the mantle on the heart rate of Helix aspersa was studied by Bagust et al. (9). Dieringer et al. (10) investigated the effects on heart rate of a noxious stimulus, in which a few salt crystals were applied to the parapodia of intact Aplysia californica submitted to cardiac denervation. Weatherill and Chase (11) studied heart activity during withdrawal reflexes in reduced preparations of $H$. aspersa.

Studies have been conducted in our laboratory to determine the effects of different external and/or internal factors and behavior on the heart rate of the terrestrial snail $M$. mogianensis. These studies have also established the relative contributions of intrinsic and neural control to heart rate $(2,3,12,13)$. Recently, we described the effects of a brief jet of water delivered to the anterior portion of the body-head on the heart rate of $M$. mogianensis (14).

The objective of the present investigation was to extend previous studies by determining both the effects of an aversive stimulus (contact with a hot plate) on the heart rate of these snails and the role of heart innervation in the mediation of such alterations.

Adult specimens (79-185 g) of M. mogianensis Simone and Leme (= M. sanctipauli sensu Romero and Hoffmann non Pilsbry and Ihering 1900) collected at Santa Rita do

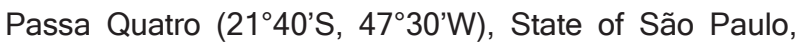
Brazil, were used. The animals were kept in a terrarium and fed green leaves ad libitum once a day.

To record heart rate, three silver chloride electrodes were implanted chronically into the visceral mass of the animals according to the technique described by Romero and Hoffmann (15). Forty-eight hours before the experiments, each animal was prepared for electrocardiogram recording and placed individually in a plastic container (29 x 29 x $14 \mathrm{~cm}$ ) containing a wet cotton patch. Animals were transferred to the experimental room kept at $25^{\circ} \mathrm{C}$, about 3 $\mathrm{h}$ before the beginning of the experiments in order to obtain a regular heart rate. Recordings were obtained with a Nihon Kohden polygraph model RM-6100 (Nihon Kohden Corporation, Tokyo, Japan).

The aversive stimulus employed in these experiments was a thermal stimulus initiated by contact with an aluminum plate mounted on the surface of a $52 \pm 1^{\circ} \mathrm{C}$ water bath (Fanem-Unitemp). The heart rate of completely extended (still or in locomotion) snails was registered for roughly 3 min. Then, each animal was placed on the hot plate and remained there until the anterior part of the head/foot lifted to a minimum of $1 \mathrm{~cm}$ (1). After this time, it was quickly removed from the thermal surface. This avoidance behavior was so vigorous that it interfered with recordings while the animal was on the hot plate and for some time after removal. Thus, the heart rate post-stimulation was recorded 60 to $90 \mathrm{~s}$ after removal of the animal from the hot plate. Heart rate data are reported as beats per min (bpm).

We first determined how the aversive stimulus caused the heart rate to vary in a group of intact animals $(N=10)$. The interference caused by the vigorous aversive response extended until $90 \mathrm{~s}$ after stimulation and prevented several measurements in several animals. For this reason, each animal was submitted to six stimulations, from which the first three legible recordings were used to calculate the post-stimulation mean value. Several animals tended to become inactive or retract into their shell after stimulation. It was necessary to spray these animals with water to keep them active or make them emerge from the shell between stimulations (14). To prevent probable anticipatory cardiac responses, a variable interval between stimulations corresponding to the time until basal heart rate recovery was employed. In order to determine the role of the nervous system in mediating these heart rate alterations, two other groups were tested: denervated animals $(N=8)$ submitted to section of the cardiac nerve (16) and sham-operated control animals ( $N=8$ ) submitted to the same surgical procedure except that the nerve was localized but left intact. Operated animals were submitted to experimental protocols 8 to 14 days after surgery. Each animal was submitted to 3 to 6 stimulations, from which the first three legible recordings were used to calculate the post-stimulation mean value. At the end of the experiments, each animal was dissected to visually confirm that the nerve had been severed. All experiments were performed between October and April.

Data from repeated measures made on the same group of animals under different conditions were analyzed statistically using a one-way analysis of variance for repeated measures followed by the Newman-Keuls comparison test. Data from two measurements made on the same group of animals were analyzed statistically by the two-tailed paired Student $t$-test, whereas comparisons between two different groups of animals were made by the two-tailed Student $t$-test. All comparisons with a probability $<0.05$ were considered to be statistically significant.

Intact snails placed on the hot plate displayed an aversive response consisting of a narrowing of all parts of the head/foot followed by a stereotyped elevation of the anterior portion associated with mucus secretion (1). All stimulated intact animals showed an increase in heart rate $(P<0.0001)$, with mean values ranging from $35.6 \pm 1.2$ 
bpm (basal heart rate) to $43.8 \pm 0.9 \mathrm{bpm}$ (post-stimulation heart rate). As shown in Figure 1, repeated stimulation did not affect the magnitude of the increase in heart rate, with values varying from $6.8 \pm 1.1$ to $8.8 \pm 0.8 \mathrm{bpm}$. Repeated stimulations did not affect the basal heart rate recovery time, whose mean values varied from $4.3 \pm 0.3$ to $5.8 \pm 0.6$ min. To exclude the possibility that stimulation with water interfered with the animals' arousal levels between aversive stimulations, statistical analysis was performed in a subgroup of 5 animals in which we could obtain sequences of three stimulations without the use of water. This analysis showed that there was no significant difference between the increase in heart rate elicited by these three stimulations. The mean heart rates corresponding to the first, second, and third stimuli were $7.9 \pm 1.3,7.9 \pm 1.7$, and 7.7 $\pm 1.3 \mathrm{bpm}$, respectively. The same finding was observed for the time until basal heart rate recovery $(4.4 \pm 1.2,5.0 \pm$ 1.0 , and $4.6 \pm 0.6 \mathrm{~min}$, respectively).

The aversive stimulus caused the heart rate to increase significantly in sham-operated (from $33.5 \pm 1.0$ to $41.2 \pm 1.3 \mathrm{bpm}, \mathrm{P}=0.0002$ ) and denervated (from $33.7 \pm$ 0.8 to $38.1 \pm 0.9 \mathrm{bpm}, \mathrm{P}<0.0001$ ) animals. Nevertheless, Figure 2 shows that the increase in heart rate obtained in denervated animals differed significantly from that obtained in sham-operated animals $(P=0.011)$. The mean increase in heart rate in denervated animals (4.4 \pm 0.4 bpm) was $57 \%$ of the value obtained in sham-operated animals $(7.7 \pm 1.3 \mathrm{bpm})$, indicating that the cardiac nerve is responsible for $43 \%$ of the increase in heart rate induced by the aversive stimulus. However, the time until heart rate recovery did not differ significantly between groups $(4.9 \pm$ $0.8 \mathrm{~min}$ in sham-operated and $4.3 \pm 0.4 \mathrm{~min}$ in denervated animals).

The stimulation of $M$. mogianensis with an aversive thermal stimulus induces a withdrawal reaction characterized by a stereotyped elevation of the anterior portion of the fully extended head/foot. This is accompanied by mucus secretion, after which the animals tend to retract into the shell (1). The present results showed that the withdrawal response is always associated with an increase in heart rate followed by a gradual recovery to the basal heart rate. Dieringer et al. (10) also reported that salt crystals applied to the parapodia of $A$. californica elicited vigorous escape activity accompanied by both mucus secretion and an increase in heart rate. In contrast, Bagust et al. (9) reported that strong mechanical stimulation of the mantle of $H$. aspersa evoked a burst of activity in the intestinal nerve accompanied by inhibition of the heart. Recently, Weatherill and Chase (11) used a reduced preparation of $H$. aspersa to observe that tactile stimulation of body organs elicited coordinated responses of the heart whose most prominent feature was excitation often preceded by transient inhibition.

The increase in heart rate during withdrawing reflexes seems to be an adaptive strategy, since the blood must deliver more oxygen to the tissues during these vigorous muscular activities. This can be achieved through an increase in cardiac output resulting from an increase in heart rate.

Our results also showed that repetition of the aversive stimulus affects neither the magnitude of the heart rate increase nor the recovery time in a series of six stimulations in intact snails. In contrast, Romero and Hoffmann

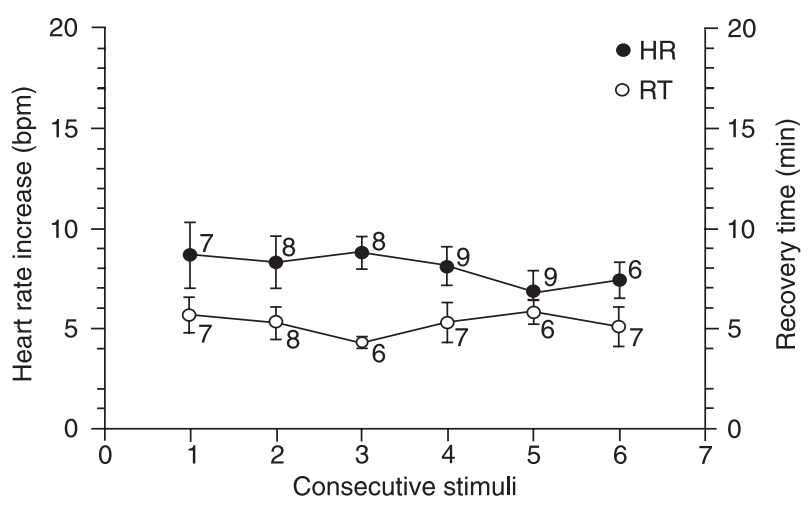

Figure 1. Effect of repeated aversive stimulation on the heart rate increase (HR, closed circles) and time until basal heart rate recovery (RT, open circles) in intact specimens of Megalobulimus mogianensis. A group of 10 snails was tested. Data are reported as means \pm SEM for 6 to 9 legible recordings as indicated to the right of each point in the figure. Heart rate data are reported as beats per minute (bpm), and recovery time is reported as $\min$ at $25^{\circ} \mathrm{C}$.

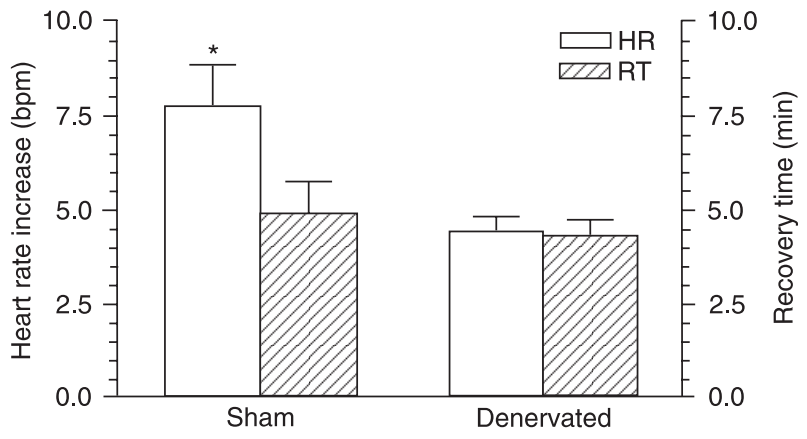

Figure 2. Heart rate (HR) increase and time until basal heart rate recovery (RT) associated with an aversive stimulus in shamoperated and denervated specimens of Megalobulimus mogianensis. Data are reported as means \pm SEM for 8 specimens in each group. Heart rate data are reported as beats per minute (bpm), and recovery time is reported as min at $25^{\circ} \mathrm{C}$. ${ }^{*} \mathrm{P}<0.05$ compared to denervated animals (Student $t$-test). 
(14) showed that repetition of an innocuous stimulus induces a gradual decrease in the magnitude of the heart rate increase in a series of three stimulations. Their findings indicate that this behavioral response undergoes early habituation, a form of learning common to all animals (7). Although a decrease in the strength of the response when a non-threatening stimulus is repeatedly presented confers an adaptive advantage to the animal by reducing energy expenditure (6), ignoring an aversive stimulus is disadvantageous since it may result in harm or even death.

Our investigations into the role of the nervous system in mediating heart rate alterations revealed that stimulation by the aversive thermal stimulus induces significant increases in heart rate in both sham-operated and denervated animals. However, the mean increase in the denervated animals was significantly lower than that observed in snails with an intact cardiac nerve. In contrast, Dieringer et al. (10) reported that although noxious stimulus induced significant increases in heart rate in both sham-operated and denervated specimens of $A$. californica, the heart rate responses were not significantly different for the two groups. Our present results showed that the cardiac nerve is responsible for $43 \%$ of the increase induced by the aversive stimulus in $M$. mogianensis. Weatherill and Chase (11) concluded that cardiac responses during withdrawal responses are mediated by the activation of central nervous circuits in $H$. aspersa. Nevertheless, these investigators could not determine the percentage of nervous system contribution to these cardiac alterations since their results originated from reduced preparations in which the venous return was maintained at a constant rate.

The nervous system simultaneously activates motor behavioral patterns and correlated vegetative adjustments. The associated neural and humoral control of bodily functions by the vegetative nervous system has likely evolved to regulate basic survival strategy in both vertebrates and invertebrates (5). Thus, the increase in heart rate triggered by neural activity represents the contribution of the nervous system to the withdrawal response induced by aversive stimulation. On the other hand, the increase in heart rate observed in denervated animals is expected since the aversive stimulus induces a vigorous narrowing of all parts of the head/foot followed by the elevation of the anterior part of the body. This intense muscular activity may generate an increase in cephalopedal hemocoel pressure and consequently venous return, which is responsible for the increase in heart rate (3). In support of this mechanism, Romero and Hoffmann (14) demonstrated a significant positive correlation between the time necessary for head retraction-protraction and the increase in heart rate in denervated snails submitted to an innocuous stimulus. Since noxious stimulation of the body wall may release stress hormones into the hemolymph of $A$. californica $(17,18)$, humoral factors may also be involved in the increase in heart rate observed in denervated snails submitted to an aversive thermal stimulus.

Although aversive stimulus induces smaller increases in heart rate in denervated animals, our results also show that the recovery time associated with these increases did not decrease compared to that of snails with intact cardiac nerves. This finding indicates that the cardiac nerve probably has a delayed inhibitory effect, which may play a role in the recovery of the basal heart rate. It is well-known that mollusk cardiac nerves, which originate from the visceral ganglion, contain inhibitory as well as excitatory fibers. In their work on intact specimens of $M$. mogianensis, Romero and Hoffmann (14) showed that the delivery of the first innocuous stimulus (a jet of water) induced a heart rate increase of $7.4 \pm 1.2 \mathrm{bpm}$ associated with a recovery time of $15.5 \pm 1.8 \mathrm{~min}$. Our present data showed a similar increase in the heart rate of snails submitted to an aversive thermal stimulus $(8.7 \pm 1.6 \mathrm{bpm})$ but a faster recovery of the basal heart rate $(5.7 \pm 0.9 \mathrm{~min})$. To promote a faster basal heart rate recovery, an inhibitory effect via the cardiac nerve is necessary. This conclusion agrees with that of S-Rósza and Salánki (19), who stated that "besides the excitatory synaptic connections, inhibitory ones may also exist to determine the time limitation of the response".

Although both innocuous and aversive stimuli induce heart rate increases of similar magnitude, it is remarkable that the recovery time associated with an innocuous stimulus is roughly three times greater than that associated with an aversive one. A jet of water, similar to the rain after the drought, has an alerting effect. It induces an increase in the general activity level, which is reflected in the tendency of snails to accelerate their movements after the withdrawal reaction (14). On the other hand, the aversive thermal stimulus may be similar to contact with a hot rock surface (20); if a stimulus of this nature persists, it may threaten the survival of a cold-blooded animal. The immediate lifting of head-foot provides snails with temporary relief from the hot surface. The ensuing fast decrease in heart rate would lead to a decrease in fluid pressure within the hemocoel, which would thereby reduce the resistance of withdrawing the foot into the shell and permit rapid retraction of the entire snail into the protective shell (9). In agreement with the above statement, we observed that several snails retracted into their shells after hot plate stimulation. 


\section{References}

1. Romero SMB, Hoffmann A, Menescal-de-Oliveira L. Is there an opiate receptor in Megalobulimus sanctipauli? Action of morphine and naloxone. Comp Biochem Physiol C 1994; 107: 37-40.

2. Romero SMB, Hoffmann A. Heart rate and behavioral patterns of Megalobulimus sanctipauli (Mollusca, Gastropoda, Pulmonata). Braz J Med Biol Res 1991; 24: 223-227.

3. Romero SMB, Hoffmann A. Role of the visceral nerve in heart rate variations during different behavioral patterns in Megalobulimus sanctipauli (Mollusca, Gastropoda, Pulmonata). Comp Biochem Physiol A 1992; 103: 93-98.

4. Krontiris-Litowitz JK. Sensitizing stimulation causes a longterm increase in heart rate in Aplysia californica. J Comp Physiol A 1999; 185: 181-186.

5. Schapker H, Breithaupt T, Shuranova Z, Burmistrov Y, Cooper RL. Heart and ventilatory measures in crayfish during environmental disturbances and social interactions. Comp Biochem Physiol A 2002; 131: 397-407.

6. Keppel E, Scrosati R. Chemically mediated avoidance of Hemigrapsus nudus (Crustacea) by Littorina scutulata (Gastropoda): effects of species coexistence and variables cues. Anim Behav 2004; 68: 915-920.

7. Frost $\mathrm{WN}$, Brandon $\mathrm{CL}$, Van Zyl C. Long-term habituation in the marine mollusk Tritonia diomedea. Biol Bull 2006; 210: 230-237.

8. Cleary LJ, Byrne JH. Identification and characterization of a multifunction neuron contributing to defensive arousal in Aplysia. J Neurophysiol 1993; 70: 1767-1776.

9. Bagust J, Fitzsimons TR, Kerkut GA. Evidence for integrating activity in the isolated intestinal nerve of Helix aspersa. Comp Biochem Physiol A 1979; 62: 397-400.

10. Dieringer N, Koester J, Weiss KR. Adaptive changes in heart rate of Aplysia californica. J Comp Physiol 1978; 123: 11-21.

11. Weatherill $D$, Chase R. Modulation of heart activity during withdrawal reflexes in the snail Helix aspersa. J Comp Physiol, A 2005; 191: 335-362.

12. Romero SMB, Hoffmann A. Heart rate and temperature in the snail Megalobulimus sanctipauli: role of the cardiac nerve. Can J Physiol Pharmacol 1996; 74: 1362-1365.

13. Rizzatti ACS, Romero SMB. Heart rate and body weight alterations in juvenile specimens of the tropical land snail Megalobulimus sanctipauli during dormancy. Braz J Med Biol Res 2001; 34: 959-967.

14. Romero SMB, Hoffmann A. Role of the cardiac nerve in the effect of a novel innocuous stimulus on the heart rate of Megalobulimus mogianensis. Braz J Med Biol Res 2006; 39: 833-837.

15. Romero SMB, Hoffmann A. A technique for recording the electrocardiogram of Megalobulimus sp. (Mollusca: Gastropoda: Pulmonata) during activity. Comp Biochem Physiol $A$ 1988; 90: 115-116.

16. Romero SMB, Hoffmann A. A technique for cardiac denervation in Megalobulimus sanctipauli (Mollusca, Gastropoda, Pulmonata). Braz J Med Biol Res 1991; 24: 1185-1188.

17. Cooper BF, Krontiris-Litowitz J, Walters ET. Humoral factors released during trauma of Aplysia body wall. II. Effects of possible mediators. J Comp Physiol B 1989; 159: 225235.

18. Krontiris-Litowitz JK, Cooper BF, Walters ET. Humoral factors released during trauma of Aplysia body wall. I. Body wall contraction, cardiac modulation, and central reflex suppression. J Comp Physiol B 1989; 159: 211-223.

19. S-Rósza K, Salánki J. The role of interneuronal connections in the regulation of heart beats in the snail Helix pomatia L. Annal Biol Tihany 1974; 41: 45-55.

20. Williams GA, De Pirro M, Leung KMY, Morritt D. Physiological responses to heat stress on a tropical shore: the benefits of mushrooming behaviour in the limpet Cellana grata. Mar Ecol Prog Ser 2005; 292: 213-224. 